developments of an initially Gaussian velocity profile are shown with solid lines in Fig. 4. Fig. 5 shows similar results for an initially triangular velocity profile (solid lines). It is observed that the initial velocity gradient is distorted due to the action of the nonlinear anomalous viscosity.

The results of Figs. 1 and 2 cannot be directly compared with those of Figs. 4 and 5 because the time scale $T$ and $\tau$ are different. From (4) and (7), it is observed that

$$
\frac{\tau}{T}=\frac{|V|}{|\partial V / \partial Y|^{2}} \text {. }
$$

In order to be able to compare the results of our linear analysis to the nonlinear ones, we must use some average of the righthand side of (10). We employ the following initial estimate:

$$
\frac{\tau}{T}=\frac{\int_{-\infty}^{+\infty}|V|_{\text {initial }} d Y}{\int_{-\infty}^{+\infty}|\partial V / \partial Y|_{\text {intial }} d Y}
$$

Substituting (8) and (9) in (11), we find that $\tau / T$ for initially Gaussian and triangular velocity profiles are $4 \sqrt{2}$ and 12.5 , respectively. The velocity profiles of linear analysis at equivalent times are also plotted in Figs. 4 and 5. It is observed that the constant anomalous viscosity destroys the velocity gradient more rapidly as compared with the nonlinear one given by (2). The decay of the center line velocity in accordance with the nonlinear theory (solid line) and the linear theory (broken line) are shown in Fig. 6 where $T$ is converted to $\tau$.

\section{REFERENCES}

[1] A. Hirose and I. Alexeff, Phys. Fluids, vol. 16, p. 1087, 1973.

[2] A. Hirose and H. M. Skarsgard, Phys. Rev. Lett., vol. 29, p. 1432, 1972.

[3] K. E. Lonngren, W. F. Ames, A. Hirose, and J. Thomas, Phys. Fluids, vol. 17, p. 1919, 1974.

[4] G. Ahmadi and A. Hirose, to be published.

[5] W. Horton, Jr., Phys. Rev. Lett., vol. 28, p. 1506, 1972.

[6] S. P. Gary and J. Schmidt, Phys. Lett., vol. 48A, p. 23, 1974.

[7] E. C. DuFort and S. P. Frankel, Matt. Tab. Wash., vol. 7, p. 135, 1953.

\title{
Absolute Calibration of a Prompt Gamma-Ray Detector for Intense Bursts of Protons
}

\author{
F. C. YOUNG, W. F. OLIPHANT, S. J. STEPHANAKIS, AND A. R. KNUDSON
}

\begin{abstract}
A detector for determining the intensity and duration of kiloampere pulsed proton beams is described. Proton intensities are inferred from measurements of prompt gamma rays of 6 to $7 \mathrm{MeV}$ from the ${ }^{19} \mathrm{~F}(p, \alpha \gamma)^{16} \mathrm{O}$ reaction. The thick-target yield for this reaction on a Teflon target was measured for $0.5-$ to $2.8-\mathrm{MeV}$ protons. Two different size detectors were calibrated absolutely using the continuous proton beam from a Van de Graaff accelerator. An absolute measurement of a kiloampere pulsed proton beam is described, and the measured current is in agreement with an independent determination of the total proton intensity by nuclear activation.
\end{abstract}

\section{INTRODUCTION}

$\mathrm{T}$ HE GENERATION of intense proton beams from highpower $\left(10^{12}-\mathrm{W}\right)$ pulsed $\left(10^{-8}-\mathrm{s}\right)$ plasma sources has required the development of new diagnostics to evaluate such

Manuscript received March 19, 1980; revised October 9, 1980. This work was supported in part by the Department of Energy and in part by the Defense Nuclear Agency.

The authors are with the Naval Research Laboratory, Washington, DC 20375. beams. Measurements [1] of intense pulsed proton beams have been carried out using nuclear activation [2], but this technique is limited for high current densities by ablation of the target being activated. Attenuating screens may be used to overcome this problem to some extent. The nuclear activation technique does provide a measure of the total number of ions.

Also, it is of interest to measure the time variation of the proton current. Such measurements have been carried out with a scintillator-photodiode detector in a time-of-flight arrangement, but it is difficult to provide a light shield on the scintillator sufficiently thin to transmit the protons and yet sufficiently robust to survive the proton intensities intact. Furthermore, magnetic or electrostatic deflection is complicated because the beam is charge neutralized. These diagnostics are even more difficult to use with the development of more intense proton beams and focused beams [3].

In this paper, a diagnostic for intense bursts of protons using prompt gamma rays from a nuclear reaction is described. The 
gamma-ray detector consists of a scintillator and photomultiplier enclosed in a lead shield. A nuclear reaction is selected which produces gamma rays of several mega-electronvolts energy. Then the lead shield will effectively attenuate the bremsstrahlung ( $E \leqslant 1 \mathrm{MeV}$ ) from the generator and will convert the multi-mega-electronvolts prompt gamma radiation into annihilation quanta and other lower-energy quanta by pair production and multiple Compton interactions in the lead shield. In this way, the shield becomes a source of lowerenergy prompt radiation which can be detected efficiently with a small plastic scintillator.

For this diagnostic the nuclear reaction ${ }^{19} \mathrm{~F}(p, \alpha \gamma){ }^{16} \mathrm{O}$ is selected. This reaction is resonant with low-energy protons $\left(E_{p} \geqslant 0.34 \mathrm{MeV}\right)$ and produces gamma rays with energies of 6 to $7 \mathrm{MeV}$. The most intense gamma ray is $6.13 \mathrm{MeV}$, but 6.92- and 7.12-MeV gamma rays are also produced [4]. No other gamma rays to which our detectors are sensitive are produced by protons with energies less than $3-\mathrm{MeV}$ incident on fluorine. Prompt gamma rays from this reaction in a Teflon target can be measured with a scintillator-photomultiplier detector of good time resolution ( $\leqslant 5 \mathrm{~ns}$ FWHM). If the detector output is calibrated absolutely, a measurement of the prompt gamma-ray response can be used to determine the total number of protons and the time variation of the proton current. This prompt measurement is unaffected by ablation of the Teflon target provided the current density is not so intense that the stopping power of protons deviates from that of a cold target [5].

Already, this diagnostic has been applied to intense proton beams using an uncalibrated detector for which only relative measurements were possible [6]. For absolute measurements, the dependence of the gamma-ray intensity on both proton energy and angle of the emitted gamma rays must be known. Since the $\left.{ }^{19} \mathrm{~F}(p, \alpha \gamma)\right)^{16} \mathrm{O}$ reaction is resonant at many energies below $2 \mathrm{MeV}$, we have measured the intensity of gamma rays from this reaction as a function of proton energy. Intensities were measured at $0^{\circ}, 45^{\circ}$, and $90^{\circ}$ for thick Teflon targets bombarded with monoenergetic protons ranging from 0.5 to $2.8 \mathrm{MeV}$ from a Van de Graaff accelerator. Then this source of gamma rays was used to calibrate absolutely two different prompt gamma-ray detectors.

\section{Description of the Detectors}

The detectors that have been calibrated absolutely contain a $6.7-\mathrm{cm}$ diameter by $5.6-\mathrm{cm}$-thick plastic scintillator ${ }^{1}$ (NE-111) and photomultiplier ${ }^{2}$ (XP-2020) sealed in a light-tight 6-mmthick aluminum housing. No optical coupling grease is used between the scintillator and photocathode. This scintillatorphotomultiplier combination is mounted in two different size lead shields. These two detectors are illustrated in Fig. 1. The small shield is $39 \mathrm{~cm}$ long by $16 \mathrm{~cm}$ in diameter. The end plates are $3.2 \mathrm{~cm}$ thick, and the center hole is $8.9 \mathrm{~cm}$ in diameter. The large shield is $55.6 \mathrm{~cm}$ long by $26.7 \mathrm{~cm}$ in diameter. The center hole is $9.5 \mathrm{~cm}$ in diameter and the end plate in front of the scintillator is $5.1 \mathrm{~cm}$ thick. Holes for the signal

\footnotetext{
${ }_{2}^{1}$ Available from Nuclear Enterprises Ltd., San Carlos, CA 94070.

${ }^{2}$ Available from Amperex Electronic Corp., Hicksville, NY 11802.
}

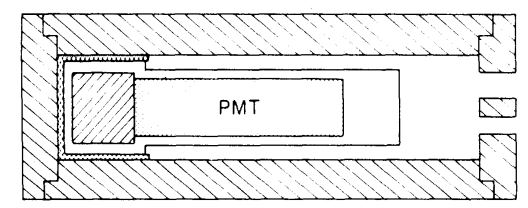

SMALL SHIELD
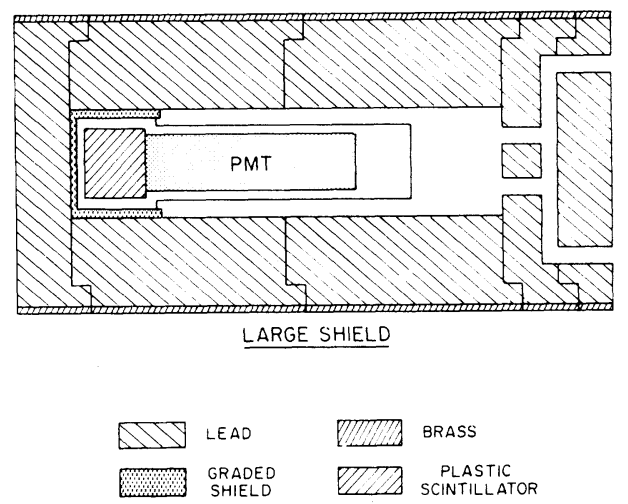

Fig. 1. Schematics of the lead shields for the small and large prompt gamma-ray detectors.

and high-voltage cables to the photomultiplier are offset in the back end plate as shown in Fig. 1. The gamma-ray source is located on the axis of the lead shield so that the scintillator is irradiated through the front end plate. A graded shield is used between the lead and the scintillator to attenuate lead K-shell $\mathrm{X}$-rays produced by the interaction of the intense bremsstrahlung in the lead shield. The graded shield consists of layers of copper $(0.13 \mathrm{~mm})$, tin $(0.38 \mathrm{~mm})$, and tantalum $(0.25 \mathrm{~mm})$. Measurements indicate that this shield attenuates the bremsstrahlung signal by about a factor of three.

\section{THICK-TARGET YIELDS}

The yield of gamma rays from proton bombardment of thick Teflon targets was measured with a $7.6-\mathrm{cm}$ diameter by $7.6-\mathrm{cm}$ thick NaI detector located $45 \mathrm{~cm}$ from the target. The targets consisted of Teflon sheet thicker than the proton range. To minimize target deterioration, beam currents of less than $0.2 \mu \mathrm{A}$ were focused into a target area $\geqslant 1 \mathrm{~cm}^{2}$. In this way, reductions in the gamma-ray yield due to target deterioration during a series of measurements were limited to less than 10 percent. The output of the NaI detector was amplified and all pulses corresponding to gamma-ray energies greater than $2.0 \mathrm{MeV}$ were counted. Under these conditions, the background in the $\mathrm{NaI}$ detector was always $\leqslant 2$ percent. At the higher energies, lesser beam currents were used to minimize pulse pile up in the NaI detector. The absolute efficiency of the NaI detector was calculated to be $8.6 \times 10^{-4}$ counts/ photon at $45 \mathrm{~cm}$ when counting only pulses greater than $2 \mathrm{MeV}$. This efficiency was calculated from interpolations of total efficiencies [7] and detector response spectra [8] for $\mathrm{NaI}$ detectors to 6.13-MeV gamma rays.

Yield measurements were normalized to the incident proton charge on target by counting the $\mathrm{NaI}$ detector output pulses for a preset integrated charge of the proton beam. The beam current and its integrated charge were measured with the Teflon target mounted in a biased Faraday cup. Yields were 


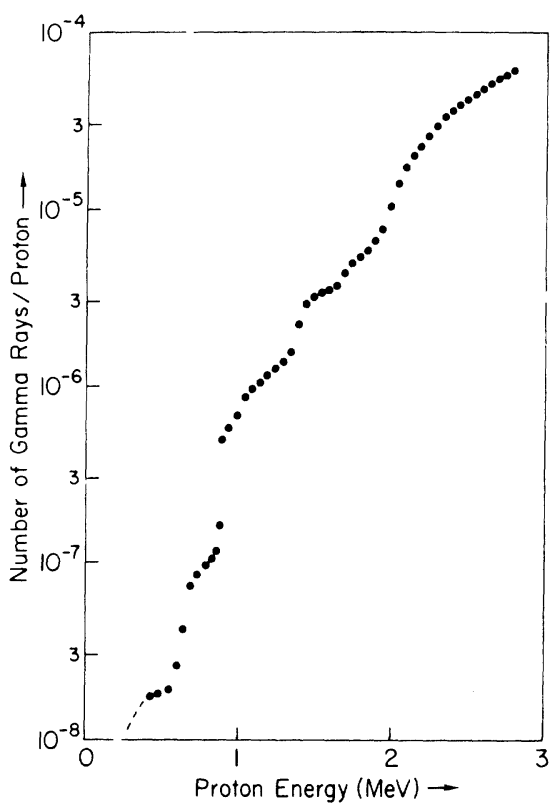

Fig. 2. Measured thick-target yield $Y(E)$ for the ${ }^{19} \mathrm{~F}(p, \alpha \gamma){ }^{16} \mathrm{O}$ reaction on a Teflon target.

measured from 0.5 - to $2.0 \mathrm{-MeV}$ proton energy in $50-\mathrm{keV}$ increments with the $\mathrm{NaI}$ detector located at $0^{\circ}, 45^{\circ}$, and $90^{\circ}$ to the incident beam direction. For energies less than $1 \mathrm{MeV}$, the $\mathrm{H}_{2}^{+}$beam from the Van de Graaff accelerator was used, and then the measurements were normalized to yields obtained at $1 \mathrm{MeV}$ with the $\mathrm{H}^{+}$beam.

Measurements at each angle were corrected for the attenuation of 6.13-MeV gamma rays in the target holder (corrections ranged from 4 to 12 percent) and compared to determine angular variations. The total angular variation at each energy was $\leqslant 10$ percent, and in fact, was $<6$ percent above the $872-\mathrm{keV}$ resonance. Since these variations are comparable to uncertainties due to target deterioration, it is concluded that no angular anisotropies are detectable in these measurements. This isotropy is not unexpected because these measurements of this nuclear reaction average over many resonances in the compound nucleus as well as over at least three independent exit channels.

Measurements at each energy were averaged over angle to provide the thick-target yields displayed in Fig. 2. The absolute energy scale is determined by the strong resonance at $872 \mathrm{keV}$ [4]. The results above $2 \mathrm{MeV}$ are based on a separate series of measurements that were carried out with a $\mathrm{NaI}$ detector located near $0^{\circ}$ and are normalized to the results below $2 \mathrm{MeV}$. It was assumed that the angular isotropy observed below $2 \mathrm{MeV}$ also applies at the higher energies. Since the gamma-ray energy spectrum is essentially constant over this range of proton energies, no corrections for variations of the detector efficiency are necessary. This is also true for the plastic scintillator gamma-ray detectors; consequently, this yield curve can be used with these detectors.

The thick-target yield has been compared to the yield reported by Stroobants et al. [9] for a composite target of $\mathrm{CaF}_{2}$ and graphite bombarded with 0.33 - to $2.16-\mathrm{MeV}$ protons. The shapes of the two yield curves are similar, however, our measurements increase somewhat more rapidly with energy. If these two yields are normalized to each other at $1.03 \mathrm{MeV}$, then our yield is 25 percent higher at $2.16 \mathrm{MeV}$ and 28 percent lower at $0.52 \mathrm{MeV}$. This difference may be due to contributions to the yield from the carbon in Teflon and from unknown impurities in the industrial grade Teflon targets. The absolute value of our yield at $1.05 \mathrm{MeV}$ is only 10 percent less than that estimated in [6] by summing the yields from many resonances below $1 \mathrm{MeV}$.

\section{Absolute Calibrations}

Calibration of both prompt gamma-ray detectors is required because the detector response is sensitive to the geometry of the lead shield. Both detectors were calibrated with the gamma source provided by the ${ }^{19} \mathrm{~F}(p, \alpha \gamma){ }^{16} \mathrm{O}$ reaction on the Van de Graaff accelerator.

The proton beam from the Van de Graaff is continuous, not pulsed, and therefore, it is necessary to relate this calibration to the detector response as recorded in pulsed-beam experiments. For measurements with intense pulsed proton beams the photomultiplier anode current is recorded on an oscilloscope which is terminated in $50 \Omega$ at the input. The measured voltage $\left(V_{d}\right)$ is related to the detector output current $\left(I_{d}\right)$ by

$$
V_{d}=(50 \Omega) I_{d} \text {. }
$$

For a continuous measurement, the detector output current is integrated in time so that a total detector charge $\left(Q_{d}\right)$ is measured for a known proton charge $\left(Q_{p}\right)$ incident on a thick Teflon target. If the ratio $\left(Q_{d} / Q_{p}\right)$ is determined for a given proton energy and target to detector distance, then the measured voltage in a pulsed experiment is related to the proton current $\left(I_{p}\right)$ at this energy by

$$
V_{d}=(50 \Omega) I_{p} Q_{d} / Q_{p} \text {. }
$$

The absolute calibration requires determining $Q_{d} / Q_{p}$ (i.e., the detector output charge for a given number of protons incident on a thick Teflon target). The dependence of $Q_{d} / Q_{p}$ on proton energy is given by the measured thick-target yield displayed in Fig. 2. This yield curve can be used to relate absolute calibrations at different proton energies and to adjust the calibrations for variations of the proton energy in experiments with pulsed proton beams.

Calibrations of both prompt gamma-ray detectors were carried out on the Van de Graaff accelerator by integrating directly the photomultiplier anode output with a current integrator $^{3}$ (Elcor, Model A310C). The detectors were located at $0^{\circ}$ to the proton-beam direction, and the measurements were carried out as a function of target to detector distance as indicated in Fig. 3. The small detector was calibrated for $1.55-\mathrm{MeV}$ protons, and the large detector was calibrated for $2.70-\mathrm{MeV}$ protons. These measurements were corrected for a background current from the photomultiplier of about $6 \mathrm{nA}$ for no proton beam. This correction amounted to 50 percent for the small detector at the largest distance, but only 4 percent for the larger detector at the largest distance. The quantity $Q_{d} / Q_{p}$ should vary as the inverse square of the distance $d$, if backscattering from the room is negligible, and may be expressed as

\footnotetext{
${ }^{3}$ Available from Elcor, Silver Spring, MD 20910.
} 


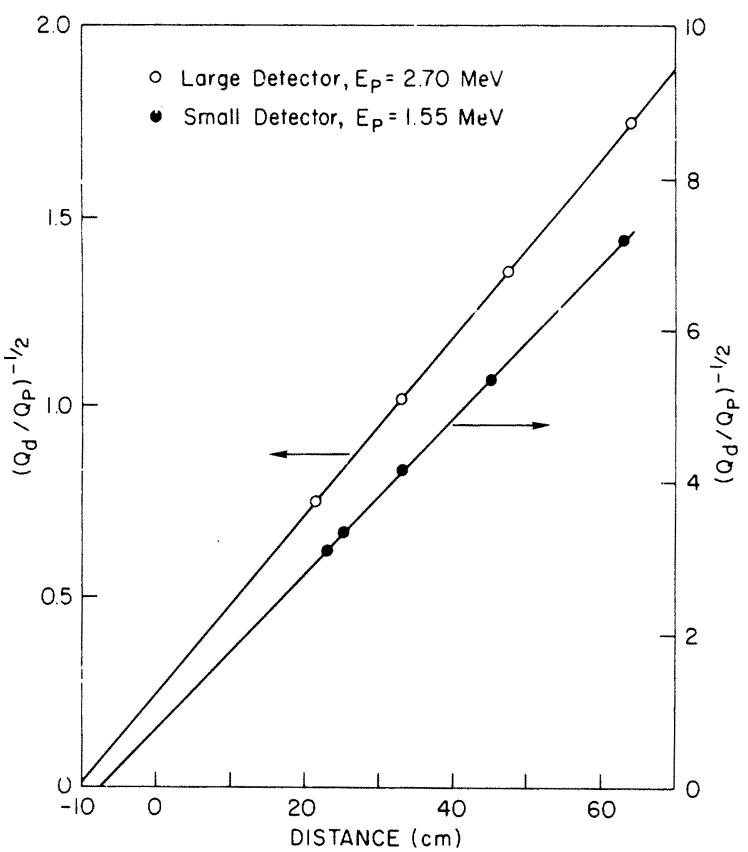

Fig. 3. Absolute calibrations of the small and large prompt gamma-ray detectors as a function of the source-to-detector distance.

$$
Q_{d} / Q_{p}=C /\left(d+d_{0}\right)^{2}
$$

where $C$ and $d_{0}$ are constants. The straight lines in Fig. 3 represent least-squares fits of (3) to the measurements. These fits correspond to $C=97 \mathrm{~cm}^{2}, d_{0}=7.8 \mathrm{~cm}$ for the small detector and $C=1810 \mathrm{~cm}^{2}, d_{0}=10.2 \mathrm{~cm}$ for the large detector.

To compare the sensitivities $C$ at large distances for these two detectors, it is noted that the thick-target yield at $2.7 \mathrm{MeV}$ is 16.3 times greater than the yield at $1.55 \mathrm{MeV}$ (see Fig. 2). Consequently, the large detector is only about 10 percent more sensitive than the small detector. The increase in sensitivity expected from the larger solid angle of the big detector is nearly compensated for by gamma-ray attenuation in the thicker lead shield of the big detector. The values of $d_{0}$ correspond to distances into the detectors which terminate inside the plastic scintillators. This suggests that gamma rays resulting from multiple interactions in lead around the circumference of the scintillator, as well as in lead in front of the scintillator, contribute to the measured detector output. The difference in $d_{0}$ values for the two detectors is due primarily to the difference in lead thicknesses in front of the two detectors. The quadratic scaling of detector output with distance indicates that the detector is relatively insensitive to roomscattered photons.

Evidence that the detector output results from the interaction of low-energy photons in the plastic scintillator is provided by pulse-height analysis of the small-detector output. A pulse-height spectrum measured for an exposure to $6.13-\mathrm{MeV}$ gamma rays is compared in Fig. 4 to a spectrum measured with a ${ }^{60}$ Co source. For the ${ }^{60} \mathrm{Co}$ spectrum, the front of the lead shield was removed and the source was placed in front of the scintillator. The high-energy cut off in this spectrum presumably corresponds to $1.1 \mathrm{MeV}$. This is the maximum energy imparted to an electron by Compton scattering of $1.3 \mathrm{-MeV}$ gamma rays in the scintillator. The high-energy cutoff in the

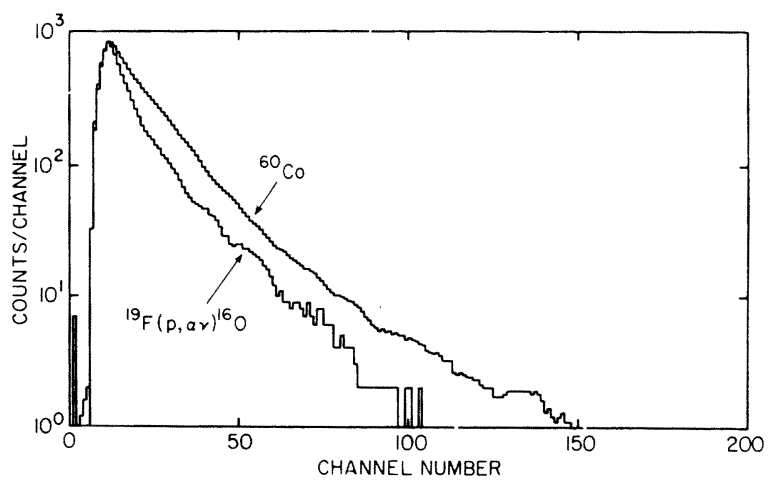

Fig. 4. Pulse-height spectra from the small prompt gamma-ray detector for the ${ }^{19} \mathrm{~F}(p, \alpha \gamma){ }^{16} \mathrm{O}$ reaction for a ${ }^{60} \mathrm{Co}$ source. The lead shield in front of the scintillator was removed for measurements with the ${ }^{60} \mathrm{Co}$ source. These spectra have been normalized at the peaks.

TABLE I

Representation of the ${ }^{19} \mathrm{~F}(p, \alpha \gamma){ }^{16} \mathrm{O}$ Thick-TARget YIELD

\begin{tabular}{|ccc|}
\hline \hline Proton Energy Range & \multicolumn{2}{c|}{$\mathrm{Y}(\mathrm{E})=\mathrm{a}+\mathrm{bE}$} \\
\hline (keV) & $\mathrm{a}(\mathrm{\gamma} / \mathrm{p})$ & $\mathrm{b}(\mathrm{Y} / \mathrm{p} / \mathrm{keV})$ \\
\hline $0-340$ & 0 & 0 \\
$340-575$ & $5.800 \times 10^{-9}$ & $2.671 \times 10^{-11}$ \\
$575-685$ & $-2.451 \times 10^{-7}$ & $4.631 \times 10^{-10}$ \\
$685-860$ & $-9.746 \times 10^{-9}$ & $2.474 \times 10^{-10}$ \\
$860-885$ & $-1.511 \times 10^{-5}$ & $1.770 \times 10^{-8}$ \\
$885-1335$ & $-1.234 \times 10^{-6}$ & $2.026 \times 10^{-9}$ \\
$1335-1435$ & $-1.740 \times 10^{-5}$ & $1.414 \times 10^{-8}$ \\
$1435-1635$ & $-2.455 \times 10^{-6}$ & $3.720 \times 10^{-9}$ \\
$1635-1885$ & $-1.511 \times 10^{-5}$ & $1.146 \times 10^{-8}$ \\
$1885-1985$ & $-6.048 \times 10^{-5}$ & $3.553 \times 10^{-8}$ \\
$1985 \quad 2785$ & $-1.155 \times 10^{-4}$ & $6.324 \times 10^{-8}$ \\
\hline
\end{tabular}

spectrum of the 6-MeV gamma rays occurs at a channel corresponding to $\sim 0.7 \mathrm{MeV}$. There is practically no contribution to the detector output for higher energy gamma rays.

\section{Application to Pulsed Proton Beams}

The calibration appropriate to pulsed proton-beam measurements is determined by substituting (3) into (2) and scaling the detector output according to the thick-target yield $Y(E)$ as displayed in Fig. 2. For the small detector, the measured voltage resulting from a proton current $I_{p}$ is given by

$$
V_{d}=1.5 \times 10^{9} Y(E)(d+7.8)^{-2} I_{p}
$$

where $V_{d}$ is in volts, $I_{p}$ is in amps, $d$ is in centimeters, and $Y(E)$ is the number of gamma rays/proton for protons of energy $E$. The corresponding expression for the large detector is

$$
V_{d}=1.7 \times 10^{9} Y(E)(d+10.2)^{-2} I_{p} .
$$

These relationships can be used to determine the proton current as a function of time from a measured prompt gamma-ray pulse, if the time dependence of the energy of the ions on the Teflon target is known. To facilitate the analysis of prompt gamma-ray measurements, the thick-target yield $Y(E)$ has been approximated by straight lines in restricted energy regions. This representation of $Y(E)$ is given in Table I. 
(a)

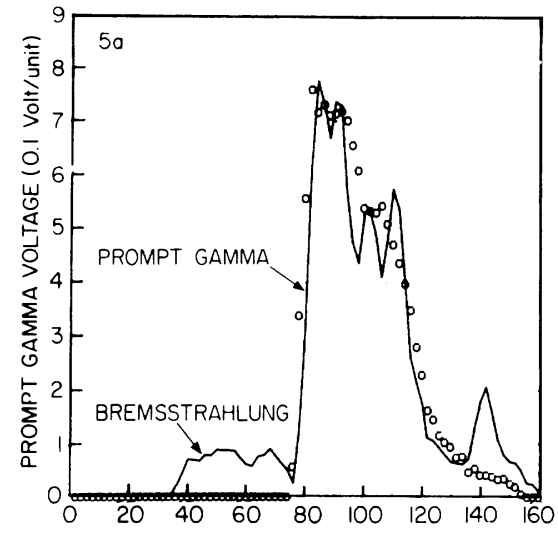

(b)

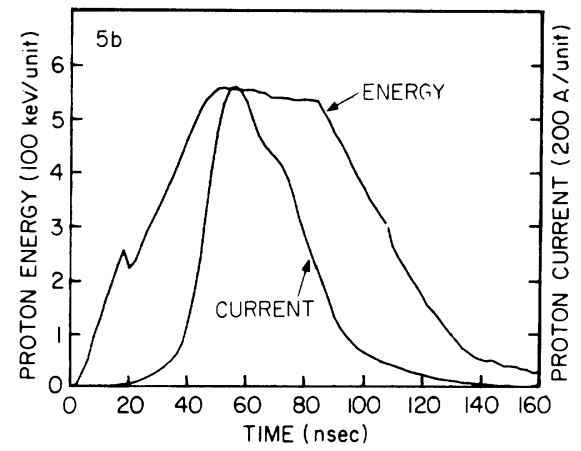

Fig. 5. The measured prompt gamma-ray detector response (solid line) in Fig. 5(a) is compared with the response (open points) calculated using the proton energy and current displayed in Fig. 5(b).

In pulsed experiments, care must be taken to insure operation of the photomultiplier in a linear regime. The detectors used in these calibrations are designed to provide linear output currents of up to $0.3 \mathrm{~A}$ at $2500 \mathrm{~V}$, which is the voltage used for the calibrations. An output of $15 \mathrm{~V}$ would be observed by a detector located $1 \mathrm{~m}$ from a Teflon target bombarded with a $1-\mathrm{MeV}, 0.13-\mathrm{kA}$ proton beam. To measure more intense proton beams, the detector sensitivity must be reduced. Optical attenuation in the form of neutral density filters may be introduced between the scintillator and photomultiplier. Also, the photomultiplier high voltage may be reduced, but this will decrease the maximum linear output voltage and reduce the fast time response of the detector.

This prompt-gamma diagnostic has been used to measure intense proton beams produced in pinch-reflex-diode experiments [3]. The proton beam is generated in a reflex-diode geometry [10] and is directed to a Teflon target located $57 \mathrm{~cm}$ from the diode. The prompt-gamma response of a small detector located $60 \mathrm{~cm}$ from the Teflon target is shown in Fig. 5(a). The prompt-gamma signal is delayed in time due to the proton time-of-flight so that the diode bremsstrahlung precedes the prompt-gamma signal. To minimize the bremsstrahlung signal in this measurement, the detector was located at $90^{\circ}$ to the ion beam direction and additional lead shielding was positioned in the line of sight between the diode and the detector.

A comparison was made of the proton current determined by the prompt-gamma measurement with the proton intensity determined from activation [2] of the carbon in the Teflon target. The proton energy is given by the measured diode voltage corrected for inductive effects. To determine the pro- ton current from the measured prompt-gamma voltage, one must correct for the flight times of different energy protons from the diode to the Teflon target. Then the time variation of the prompt-gamma voltage $V_{d}\left(t_{d}\right)$ is related to the time variation of the proton current $I_{p}(t)$ in the following way:

$$
V_{d}\left(t_{d}\right)=K \int_{0}^{\infty} Y[E(t)] I_{p}(t) \delta\left[t_{d}-t-l / v(t)\right] d t .
$$

The quantity $K$ is a constant specified by the detector geometry and the target-to-detector distance; $l$ is the proton flight path from the diode to target, and $v$ is the velocity of protons of energy $E=\frac{1}{2} m_{p} v^{2}$ emitted at time $t$ from the diode. In practice, the proton current is determined by iterating on (6) until the prompt-gamma response calculated with an assumed proton current agrees with the measured prompt-gamma response. An initial proton current is determined from a simple analytic model [1]. The final proton current and energy used to calculate the prompt-gamma voltage presented in Fig. 5(a) are shown in Fig. 5(b). The shape of this ion current is similar to that measured with a Rogowski coil in experiments at higher power levels [10]. For the present measurement, attenuating screens were used between the diode and the target to minimize target ablation. The current in Fig. 5(b) is that produced in the diode but attenuated by the screens.

The activation of carbon in the Teflon by the ${ }^{12} \mathrm{C}(p, \gamma){ }^{13} \mathrm{~N}$ reaction was calculated and compared with the measured number of radioactive ${ }^{13} \mathrm{~N}$ nuclei. The thick-target yield for this reaction can be expressed by the following sum over resonances

$$
Y(E)=\sum_{i=1}^{2} C_{i}\left\{\arctan \left[2\left(E-E_{i}\right) / \Gamma_{i}\right]+\arctan \left(2 E_{i} / \Gamma_{i}\right)\right\}
$$

The resonance energies and widths are taken from the literature [11].

$$
\begin{array}{ll}
E_{1}=457 \mathrm{keV}, & \Gamma_{1}=36 \mathrm{keV} \\
E_{2}=1699 \mathrm{keV}, & \Gamma_{2}=67 \mathrm{keV} .
\end{array}
$$

The strengths of the resonances are determined from the measurements of Seagrave [12] for carbon targets.

$$
\begin{aligned}
& C_{1}=2.41 \times 10^{1013} \mathrm{~N} / \text { proton} / \text { radian } \\
& C_{2} / C_{1}=1.44 .
\end{aligned}
$$

To convert from a carbon (C) to a Teflon $\left(\mathrm{CF}_{2}\right)$ target, the ratio of the stopping cross sections $(\epsilon)$ for $0.5-\mathrm{MeV}$ protons was determined from the literature [13]. Then $Y_{\mathrm{CF}_{2}}=$ $\left[\epsilon(\mathrm{C}) / \epsilon\left(\mathrm{CF}_{2}\right)\right] Y_{C}=0.28 Y_{C}$. The number of ${ }^{13} \mathrm{~N}$ nuclei produced in a Teflon target by the proton current and voltage displayed in Fig. $5(\mathrm{~b})$ is $(4.7 \pm 0.7) \times 10^{4}$. This uncertainty includes propagating a 10 -percent uncertainty in the thicktarget yield from Teflon and a 5-percent uncertainty in the ion voltage. A measurement of the positron decay of ${ }^{13} \mathrm{~N}$ indicates that $(5.9 \pm 0.6) \times 10^{4}{ }^{13} \mathrm{~N}$ nuclei were produced in the Teflon target. The ion energy in this measurement is small enough that no correction for deuterons in the proton beam is necessary [2]. These independent determinations of the number of 
${ }^{13} \mathrm{~N}$ nuclei are in agreement within the experimental uncertainties. It should be noted that the prompt gamma measurement only samples the proton current above $340 \mathrm{keV}$ and the carbon activation only samples the current above $\sim 440 \mathrm{keV}$. These measurements are insensitive to the current profile for proton energies below these thresholds.

\section{CONCLUSIONS}

A detector for measuring the current of intense bursts of protons with a time resolution $\sim 5$ ns has been described. Two different size detectors have been calibrated using the ${ }^{19} \mathrm{~F}(p, \alpha \gamma){ }^{16} \mathrm{O}$ reaction. The thick-target yield for this reaction on a Teflon target has been measured. A determination of the absolute proton current in a pulsed beam experiment with this diagnostic is in agreement with an independent determination of the total proton intensity in the same experiment.

\section{ACKNOWLEDGMENT}

The authors wish to thank D. Mosher and G. Cooperstein for their support and encouragement in carrying out these calibrations, B. Willis for the operation of the Van de Graaff accelerator, and J. Golden for providing the large lead shield.

\section{REFERENCES}

[1] S. J. Stephanakis, D. Mosher, G. Cooperstein, J. R. Boller, J. Golden, and S. A. Goldstein, "Production of intense proton beams in pinched-electron-beam diodes," Phys. Rev. Lett., vol. 37, no. 23, pp. 1543-1546, Dec. 1976.
[2] F. C. Young, J. Golden, and C. A. Kapetanakos, "Diagnostics for intense pulsed ion beams," Rev. Sci. Instrum., vol. 48, no. 4, pp. 432-443, Apr. 1977.

[3] D. Mosher, G. Cooperstein, S. J. Stephanakis, S. A. Goldstein D. G. Colombant, and R. Lee, "Intense focussed ion beams and their interaction with matter," in Proc. 2nd Int. Topical Conf. on High Power Electron and Ion Beam Res. and Tech. (Cornell Univ., Ithaca, NY), vol. 1, pp. 257-274, Oct. 1977; and S. A. Goldstein, G. Cooperstein, R. Lee, D. Mosher, and S. J. Stephanakis, "Focusing of intense ion beams from pinched-beam diodes," Phys. Rev. Lett ., vol. 40, no. 23, pp. 1504-1507, June 1978.

[4] F. Ajzenberg Selove, "Energy levels of light nuclei A=18-20," Nucl. Phys., vol. A300, no. 1, p. 179, May 1978.

[5] T. A. Mehlhorn, "A finite material temperature model for ion energy deposition in ion-driven ICF targets," Sandia National Lab. Tech. Rep. SAND80-0038, May 1980.

[6] J. Golden, R. A. Mahaffey, J. A. Pasour, F. C. Young, and C. A. Kapetanakos, "Intense proton beam current measurement via prompt $\gamma$ rays from nuclear reactions," Rev. Sci. Instrum., vol. 49 , no. 10 , pp. $1384-1387$, Oct. 1978.

[7] R. L. Heath, "Scintillation spectrometry," in 2nd Ed., Phillips Petroleum Co., Tech. Rep. IDO-16880, Aug. 1964.

[8] W. F. Miller and W. J. Snow, "Energy loss spectra for gamma rays in NaI," Rev. Sci. Instrum., vol. 31, no. 1, pp. 39-45, Jan. 1960.

[9] J. Stroobants, F. Bodart, G. De Conninck, G. De Mortier, and G. Nicolas, "Analysis of fluorine by nuclear reactions and application to human dental enamel," in Ion Beam Surface Layer Analysis, vol. 2, O. Meyer, G. Linker and F. Kappeler. Eds. New York: Plenum, 1967, pp. 933-943, and private communication.

[10] G. Cooperstein, "Recent advances in pinch beam ion diode research for inertial confinement," presented at the IEEE Inter. Conf. on Plasma Science (Montreal, Canada, June 5, 1979).

[11] F. Ajzenberg-Selove, "Energy levels of light nuclei A=13-15," Nucl. Phys., vol. A268, no. 1, p. 46, Sept. 1976.

[12] J. D. Seagrave, "The thick target yield of the reaction ${ }^{12} \mathrm{C}(p, \gamma)$ ${ }^{13} \mathrm{~N}\left(\beta^{+}\right)^{13} \mathrm{C}$," Phys. Rev., vol. 84, no. 6, pp. $1219-1221$, Dec. 1951.

[13] H. H. Anderson and J. F. Ziegler, The Stopping Powers and Ranges of Ions in Matter, vol. 3. New York: Pergamon, 1977.

\title{
Some Arcing Mechanisms in Lightning Protectors at Atmospheric Pressure
}

\author{
EOIN W. GRAY, SENIOR MEMBER, IEEE AND LEE G. McKNIGHT
}

\begin{abstract}
Some aspects of discharges occurring between carbon and carbon-copper electrodes in lightning protector blocks are examined. Various mechanisms that can cause arc transfer from high- to low-resistivity cathodes are considered. High resistivity in the cathode spot is a necessary but not sufficient property to cause arc motion. Diffusion of the arc plasma from an initial carbon-carbon arc to initiate a carboncopper arc appears to be the most likely means for the transfer mechanism above about $30 \mathrm{~A}$. Below $30 \mathrm{~A}$ the probability of arc transfer is considerably reduced. Effects of ac currents on these protectors are ex-
\end{abstract}

Manuscript received August 4, 1980; revised November 17, 1980.

E. W. Gray is with Bell Telephone Laboratories, Columbus, OH 43213.

L. G. McKnight is with Bell Telephone Laboratories, Murray Hill, NJ 07974. amined, and lifetimes have been predicted to within a factor of $<2$ of the experimental values.

\section{INTRODUCTION}

$\mathrm{T}$ HE CARBON BLOCK voltage arrestor is by far the commonest of the station protection devices used in the telephone service. It consists of two carbon blocks separated by a narrow air gap. High-voltage surges spark across the gap and are shorted to ground.

Due to the relatively small gap (approximately 0.003 in) and construction of the device, arc erosion products can bridge, or partially bridge, the gap and cause intermittent electrical shorts 